\title{
Effect of Magnifications of Images on Automatically Measured Grain Size
}

\author{
Kunio ITO \\ Department of Materials Science, Faculty of Engineering, The University of Tokyo, Bunkyo-ku, Tokyo, 113 Japan. \\ (Received on November 29, 1989; accepted in the final form on February 16, 1990)
}

\begin{abstract}
Grain size numbers of micrographs of ferritic steels measured by commercial automated image analyzers as well as by a newly developed program for personal computer were examined as a function of number of grains counted in a processing field. The dispersion of results due to differences in the commercial equipments has been found to be minimized when about 100 grains are taken into the field of $400 \times 400$ pixels. Comparison of values obtained by the new program with those measured manualiy has lead to the conclusion that the number of grains in the field of $400 \times 400$ pixels should be limited to not less than 100 but not more than 300 in order to find a good agreement between the two procedures.
\end{abstract}

KEY WORDS: grain size; image processing; image analysis; automatic measurement.

\section{Introduction}

The Grain Size Measurement Group of Committee on Material Evaluation by Image Analysis, Basic Research Association on Specific Subjects of The Iron and Steel Institute of Japan carried out measurements of the same photographs of particles and crystal grains by several members of the Group using automated image analysis equipments and found that the dispersion of reported values due to difference in the used equipments and algorithms were relatively large. ${ }^{1)}$

Some examples of the results are shown in Fig. 1, where grain size numbers (abbreviated as GSN's hereafter) of standard figures prepared for the comparison procedure of ferritic steel by JIS are measured by the planimetric or Jeffries' procedure. There are 4 groups of 6 bars in the figure. A group corresponds to a figure and a bar stands for a member. Numerals under the abscissa indicate averaged values of the six observers. The values $2.4,4.7,6.9$, and 7.8 correspond respectively to results of the standard figures for GSN 2, 5, 7, and 8. Height of the bars, $\Delta \mathcal{N}$, presents difference between the averaged values and values of each observer. Fig. 1 indicates that the

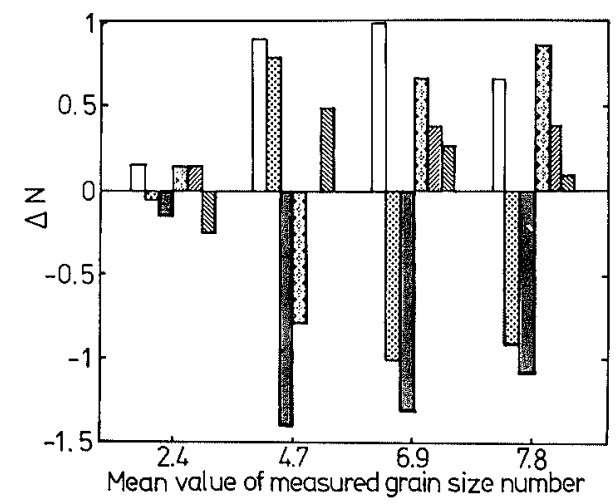

Fig. 1. Differences among commercial image analyzers presented by obtained grain size numbers. maximum difference in the 6 observers for a standard figure amounts to 2.5, although the evaluation was done after repairing images manually in the image analyzers. The difference is, however, small, when a number of grain in a field is small, i.e., in the case of the figure of the lowest GSN.

The Grain Size Measurement Group determined hence to develop a new computer program to measure automatically grain size of single phase materials by personal computer and at the same time to examine further effect of numbers of grains in a field on obtained GSN in order to minimize the dispersion of results due to difference in the equipments and algorithms used by the members of the Group.

The present paper reports principal results of the examination and of the new program tested in the same viewpoint. Characteristic algorithms of the program and effect of sample preparation on the quality of images processed by the program will be reported elsewhere. ${ }^{2-4)}$

\section{Experimentals}

\subsection{Measurement of Schematic Grain Boundary Networks}

GSN's of model networks assumed to correspond to GSN 5 and shown in Fig. 2 were determined by the members using image analysis equipments with the planimetric procedure and presented in Fig. 3, where there are pairs of $\Delta \mathcal{N}(\mathbf{m})$ and $\sigma(\square)$. The 1st to 3 rd pairs from the left side are the results of Fig. 2(a), and the 4th to 6th are those of Fig. 2(b). The 7th to 9 th, and the 10 th to 12 th correspond respectively to Figs. 2(c) and 2(d). The three pairs belonging to each network represent results obtained by 3 magnifications by which the network pattern was taken into the image analyzers. The size of a field was there fixed to be composed of $400 \times 400$ pixels.

Numerals under the abscissa show average numbers of grains counted in one field. The height of bars, $\Delta \mathcal{N}$ presents differences between the average of 
Fig. 2.

Schematic grain boundary network models.


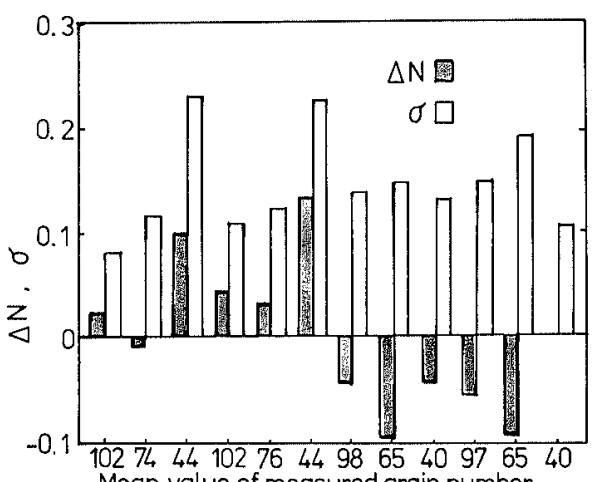

Fig. 3. Results of the schematic network models measured by commercial image analyzers.

GSN's reported by 9 observers for each magnification and for each network and the average of the all (4 networks $\times 3$ magnifications $\times 9$ observers) measurements, while $\sigma$ shows the corresponding standard deviations of the 9 observers. Values of $\Delta \mathcal{N}$ are positive, when the network is completely connected. They are, however, negative, for disconnected net works, resulting from the fact that repairs of the disconnection by the used equipments are in most case incomplete. The $\sigma$ values increase with decreasing numbers of grains in a field (density of grains), when the networks are complete. This must be attributed on the one side to the statistical reason. This is on the other side due to the fact that some of the used algorithms regard grains on the border line of measuring field as not half but whole grains, while the other orthodox algorithms leave them out of counting. When a grain on the border line is counted as not a half but unity, an obtained GSN appears to be larger than it should be. Average GSN's obtained by counting about 100 grains in a field are in Fig. 3 smaller than those calculated from about 40 grains. This re- sult corresponds also to the border line effect, because the effect is the more distinct, the smaller the number of counted grains is.

It is generally observed in experiments done by the Grain Size Measurement Group that the repairs of disconnected grain boundaries are the better, the smaller the number of grains in a field is, although Fig. 3 does not clearly show this tendency. It is likely that the repairs of Figs. 2(c) and 2(d) by the used algorithms might have been satisfactory only in the case of the smallest number of grains in a field.

\subsection{Measurement of Ferritic Steels}

Two low carbon steels were taken by 2 magnifications (photo-magnification in Table 1) for each steel. Keys beginning with letters $\mathrm{A}$ and $\mathrm{B}$ in Table 1 correspond to the steels and representatives of the micrographs are shown in Fig. 4. They were further taken into image analyzers by 3 magnifications (cameramagnification in Table 1). The size of a field of all image analyzers were fixed to $400 \times 400$ pixels. Conditions and some fundamental results of the analysis are summarized in Table 1, where grains/field and interceptions/field are average numbers counted in a field.

Results of the planimetric procedure are presented in Fig. 5(a), where the abscissa represents the conditions of analysis, corresponding to the conditions A21, $\mathrm{A} 22, \ldots$ for steel $\mathrm{A}$, and $\mathrm{B} 11, \mathrm{~B} 12, \ldots$ for steel $\mathrm{B}$ in Table 1. Ten (or 9) micrographs were distributed for each steel for each photomagnification to members of the Group, so that each member measured 10 (or 9) fields for each condition. A GSN of an observer for one measuring condition was defined as the average of 10 (or 9) fields. GSN's of the specimen A and $B$, averages of 11 observers, are obtained for each condition as 7.5 plus values presented by the mark $\square$ and as 5.0 plus those presented b the mark $\Delta$, 


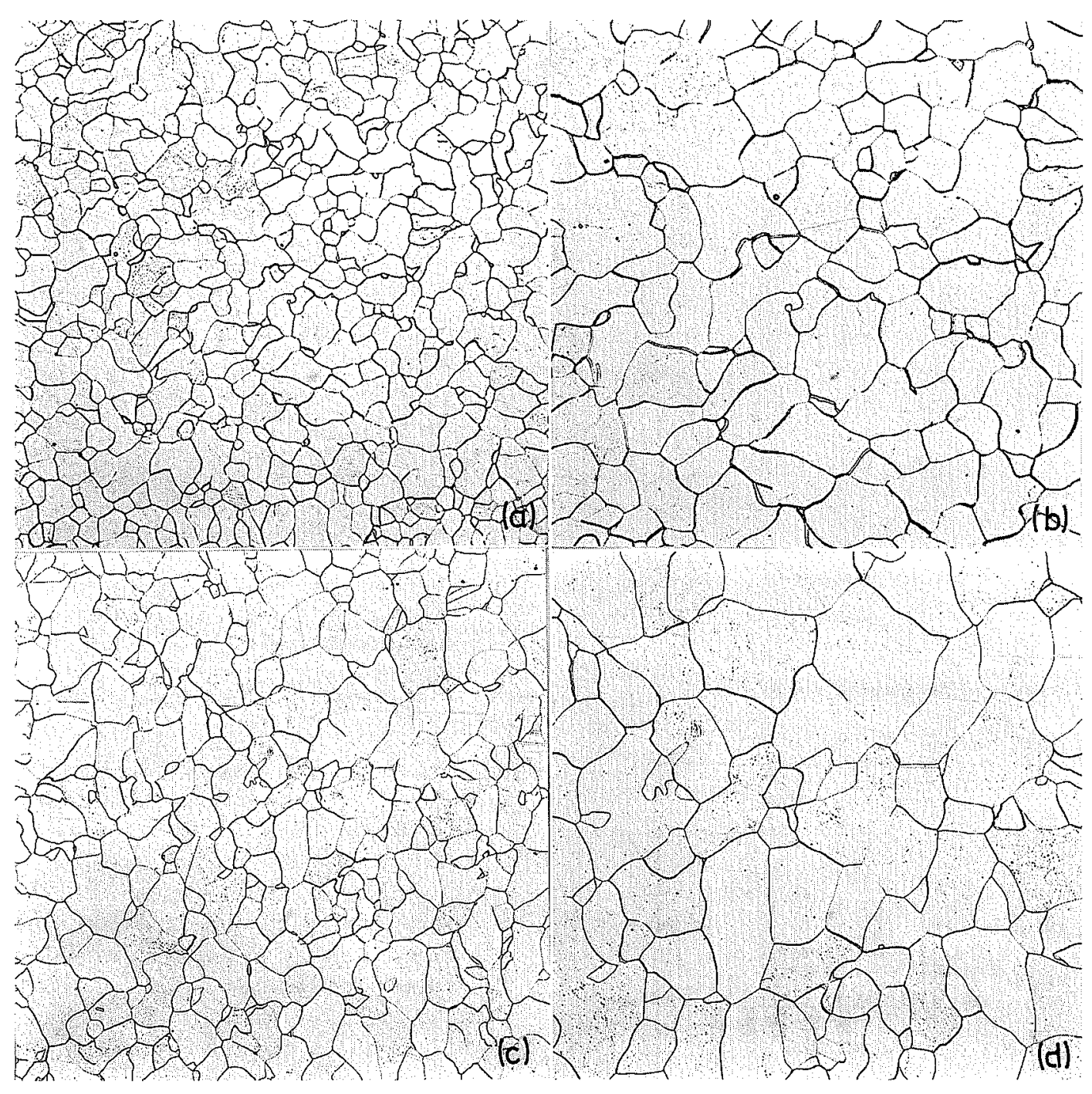

(a) Steel A ( $\times 200)$

(b) Steel A ( $\times 400)$

(c) Steel B $(\times 100)$

(d) Steel B $(\times 200)$

Fig. 4. Examples of micrographs of ferritic steels. $(\times 16 / 25)$

Table 1. Analyzing conditions for Fig. 5.

\begin{tabular}{lrrrrrrrrrrrr}
\hline & \multicolumn{10}{c}{ Key } \\
\hline & A21 & A 22 & A23 & A 41 & A 42 & A43 & B 11 & B 12 & B 13 & B 21 & B 22 & B 23 \\
\hline Photo-magnification & $\times 200$ & $\times 200$ & $\times 200$ & $\times 400$ & $\times 400$ & $\times 400$ & $\times 100$ & $\times 100$ & $\times 100$ & $\times 200$ & $\times 200$ & $\times 200$ \\
Camera-magnification & 1 & $\sqrt{2}$ & 2 & 1 & $\sqrt{2}$ & 2 & 1 & $\sqrt{2}$ & 2 & 1 & $\sqrt{2}$ & 2 \\
Length of pixel $(\mu \mathrm{m})$ & 1.375 & 0.972 & 0.688 & 0.688 & 0.486 & 0.344 & 2.750 & 1.945 & 1.375 & 1.375 & 0.972 & 0.688 \\
Grains/Field*1 & 389.0 & 202.0 & 93.0 & 102.0 & 45.0 & 21.0 & 224.0 & 117.0 & 58.0 & 62.0 & 29.0 & 13.0 \\
Interceptions/Field*2 & 43.9 & 33.6 & 22.2 & 22.9 & 17.3 & 11.5 & 34.3 & 25.3 & 17.3 & 19.4 & 13.0 & 9.0 \\
\hline
\end{tabular}

$* 1 \quad$ For Jeffries' procedure $* 2$ For Heyn's procedure

respectively.

The dispersion of GSN on the same steel due to difference in fields was at first presented by the standard deviation [=field dispersion] of 10 (or 9) fields measured by the same observer, while the dispersion of GSN of the same field due to difference in observers was at first presented by the standard deviation [ $=\mathrm{ob}-$ server dispersion] of the 11 reported values of the same micrograph. The dispersion due to field $\left(\sigma_{\text {field }}\right)$ was then defined by average of the field dispersions of the 11 observers, while the dispersion due to observer $\left(\sigma_{\mathrm{obs}}\right)$ was defined by average of the observer dispersions of 10 (or 9) fields. The dispersion $\sigma_{\text {field }}$ and $\sigma_{\mathrm{obs}}$ are shown for the specimen $\mathrm{A}$ respectively by $\diamond$ and $\diamond$ in Fig. 5(a), and those for the specimen $\mathrm{B}$ correspondingly by $\nabla$ and $\boldsymbol{\nabla}$.

Results of the intercept or Heyn's procedure are shown in Fig. 5(b). The average GSN's of the specimen $\mathrm{B}$ are obtained as 4.5 plus values presented by the mark $\Delta$. The other marks and notation are the same as Fig. 5(a).

The following two points are indicated by Figs. 5 (a) and 5(b):

(1) The dispersion dü to observer (i.e., due to 
difference in equipments and algorithms) is larger than that due to field, so long as measured numbers of grains (or interceptions) in a field are larger than about 20.

(2) About 100 grains should be taken into one field $(400 \times 400$ pixels) of image analyzers so that the dispersion due to observers might be minimized. Under this condition, the photo-magnification should be as large as possible.

The GSN's determined by the planimetric proce-
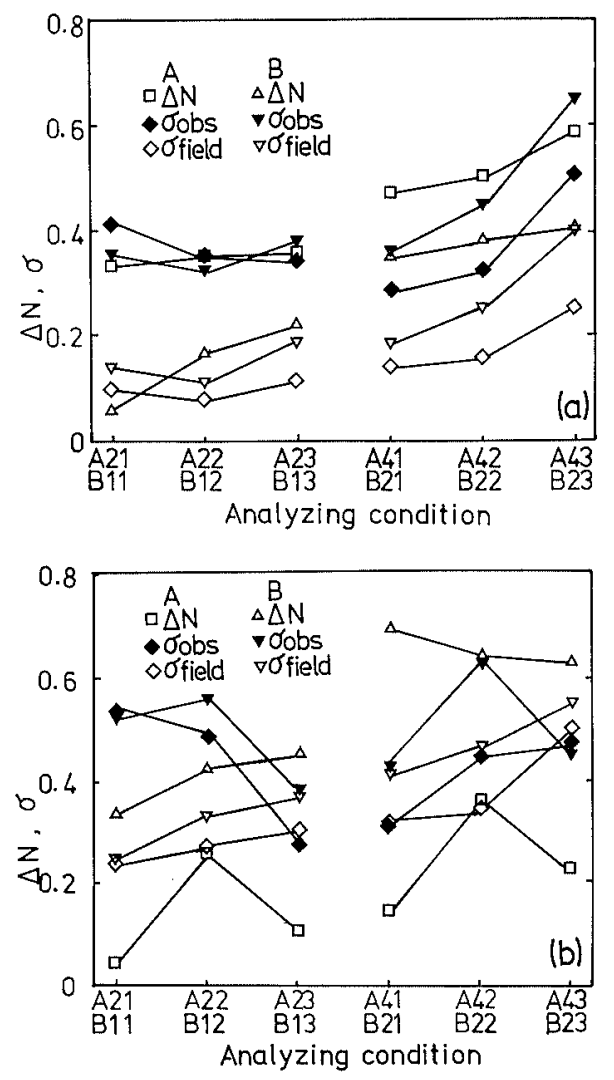

$\Delta \mathcal{N}^{\prime}$ 's: Averages of grain size numbers from which constants, 7.5 for steel $A$ in (a) and (b), 5.0 for steel $B$ in (a), 4.5 for steel $B$ in (b), are subtracted.

Fig. 5. Results of the ferritic steels measured by commercial image analyzers with the planimetric (a) and intercept (b) procedures. dure have been found in this examination to be by 0.2-0.3 larger than those determined by the intercept procedure.

\subsection{Measurement by the New Program}

The same micrographs used in the preceding section were processed and measured by the newly developed program using the planimetric as well as intercept procedures and compared with results of the same area in the same micrograph obtained manually by the planimetric procedure (Table 2). The size of processing field was fixed to $400 \times 400$ pixels in this program and almost the whole area of each micrograph in Fig. 4 was taken into the field. The abscissa and ordinate of Fig. 6 present manually (by hand) and automatically (by personal computer) measured GSN's, respectively. An open mark corresponds to a micrograph and filled marks present averages of 10 (or 9) micrographs. Differences between automatically and manually measured GSN's of the same micrographs are plotted in Fig. 7 as a function of number of grains counted in a field, i.e., against density of grains.

There are principally 2 reasons why automatically and manually measured values are different.

(1) The program can misjudge non grain boundary contrasts (for example, surface etching contrasts due to incomplete polishing, polishing scratches, second phase particles) as grain boundary contrast during binarization process, leading to the overcounting; the GSN determined by the program happens to be larger than that determined manually.

(2) The program can overlook weak etched or thin grain boundaries on binarization and discard fragments of grain boundaries as "noise" on image processing as well as on repairing network logically, leading to the undercounting.

Plotted points should fall on the oblique straight line in Fig. 6, when automatically measured values agree completely with manually measured ones. The points in Fig. 6 show rather weak correlation among the values determined by the two processes, corresponding to the fact that the overcounting and undercounting are likely to occur together even in one field.

Table 2. Comparison between procedures for grain size measurement.

\begin{tabular}{|c|c|c|c|c|c|c|c|c|c|c|}
\hline \multirow{5}{*}{ Sample } & \multicolumn{10}{|c|}{ Procedure } \\
\hline & \multicolumn{7}{|c|}{ By new program } & \multicolumn{3}{|c|}{ Manual } \\
\hline & \multirow{2}{*}{\multicolumn{3}{|c|}{ Intercept }} & \multicolumn{4}{|c|}{ Planimetric } & \multicolumn{3}{|c|}{ Planimetric } \\
\hline & & & & \multicolumn{2}{|c|}{$\begin{array}{l}\text { Grains on border } \\
\text { line not counted }\end{array}$} & \multicolumn{2}{|c|}{$\begin{array}{l}\text { Grains on border } \\
\text { line counted }\end{array}$} & \multicolumn{3}{|c|}{ Grains on border line counted } \\
\hline & GSN & STD & DIF & GSN & STD & GSN & STD & GSN & STD & DGF \\
\hline$B \times 200$ & 5.6 & 0.28 & 64 & 5.7 & 0.23 & 5.4 & 0.14 & 5.1 & 0.14 & 70 \\
\hline$A \times 400$ & 8.2 & 0.13 & 55 & 8.0 & 0.17 & 7.8 & 0.13 & 7.8 & 0.12 & 112 \\
\hline$B \times 100$ & 5.1 & 0.18 & 73 & 4.9 & 0.10 & 4.7 & 0.07 & 4.7 & 0.11 & 208 \\
\hline$A \times 200$ & 7.7 & 0.20 & 79 & 7.4 & 0.09 & 7.2 & 0.09 & 7.4 & 0.07 & 335 \\
\hline
\end{tabular}

GSN : Average grain size number

STD : Standard deviation due to dispersion among fields Ten and 9 fields are measured for steels $A$ and B, respectively.
DIF : Average number of interceptions counted in a field DGF : Average number of grains counted in a field 


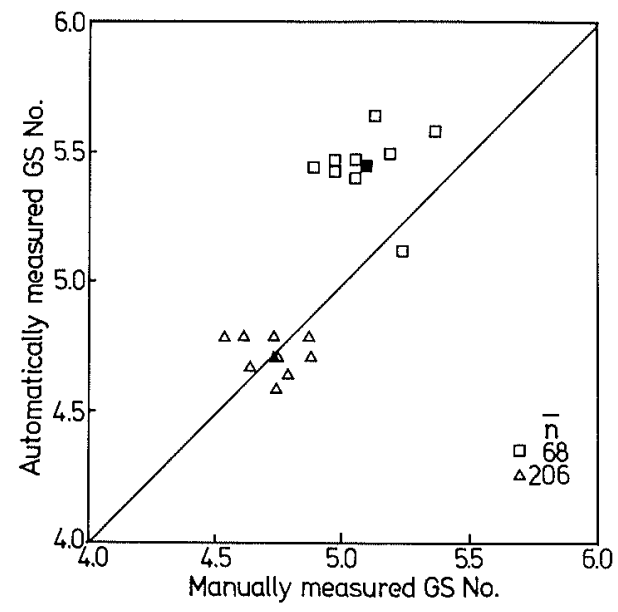

Examples of micrographs whose results are shown by $\triangle$ and $\square$ are shown in Figs. 4 (c) and 4 (d), respectively.

$\bar{n}$ : Average numbers of grains counted in a field

Fig. 6. Comparison between grain size numbers measured by the new program and hand with the planimetric procedure, grains on the border line counted.

They cancel, however, to each other on averaging over several fields so that the average of automatically measured values agrees well with that of manually measured ones so long as density of grains is in the appropriate range, i.e., between about 100 and 300, as is demonstrated by Fig. 7 .

Fig. 7 indicates further that the overcounting prevails against the undercounting, when the density of grains is too small, and vice versa. A characteristic reason of this program why degree of the over and undercounting depends on the density of grains is found in its binarization algorithm. The program binarizes pixel to pixel, using as criterion average brightness of pixels in a moving local area. When the density of grains is small, numbers of pixels of grain boundary in the local area is also small so that pixels of non grain boundary are likely to be binarized into the same contrast side as those of grain boundary. Pixels of less contrasted grain boundary are on the other hand likely to be classified into the non grain boundary contrast side, when the density of grains is large.

GSN's measured at higher magnifications of micrographs are larger than those measured at lower magnifications ( $c f$., Fig. 6 and Table 2), even if measured manually. This is attributable to decrease of overlooking of finer grain boundaries with increasing magnification. The difference is larger, when measured automatically, because of the appropriate range of magnification due to the appropriate range of density of grains ( $c f$. . Fig. 7).

The GSN obtained by this program with the planimetric procedure is found to be by $0.2-0.3$ larger (Table 2) when only grains completely included in the field are counted, than when grains on the border line of the field are also counted. If grain boundaries between grains on the border and those in the field are "overlooked" by the program, the algorithm based on ASTM E112 1984 overestimates an



Fig. 7. Difference between grain size numbers measured by the same ways as Fig. 6 and plotted against density of grains, $\Delta \mathcal{N}=$ Program-measured value - Handmeasured value.

average grain area. This must be the reason for the disagreement. The algorithm to count only grains included in the field should be adopted for application of this program with the planimetric procedure. The GSN's determined by the intercept procedure are in Table 2 by about 0.2 larger than those obtained by the planimetric procedure, being the inverse of the results presented in the preceding section. The reason is not clear.

\section{Summarizing Gonclusions}

Effects of numbers of grains in a field on grain size numbers determined by commercial as well as newly developed automated image analyzers have been examined.

(1) When each user of commercial equipments reports an average of about 10 fields for a specimen, the dispersion of the reported values from about 10 observers presented by the standard deviation is about 0.4 , while the dispersion due to the fields of the same specimen is about 0.2 .

(2) Incomplete repairing of disconnected grain boundary networks is one of the reasons for the dispersion due to the equipments. The repairs become more complete with decreasing number of grains in a field.

(3) The dispersion due to observers is minimized as a balance between completeness of repairs and statistical scatter, when about 100 grains are taken into a field of $400 \times 400$ pixels.

(4) The number of grains in a field $(400 \times 400$ pixels) has been revealed on application of the developed program to be controlled to as possible as small but not less than 100 .

(5) When about 10 fields are averaged under this condition, differences between grain size numbers obtained by the new program and those measured manually are less than 0.2 .

(6) Only grains included completely in the field should be counted on application of the new program with the planimetric procedure. 


\section{Acknowledgments}

The main part of this paper was made as cowork of the members of the Grain Size Measurement Group of Committee on Material Evaluation by Image Analysis, Basic Research Association on Specific Subject of ISIJ. The author acknowledges them, specially Mr. Shoji Terasaka, NKK Corp., for preparationof micrographs. He thanks Miss M. Fujita, The University of Tokyo, for assistance in the experiment.

\section{REFERENCES}

1) Recent Development of Image Analyzing Technology for Microstructure of Metal, Comm. on Material Evaluation by Image Analysis, Basic Research Assoc. on Specific Subjecs of The Iron and Steel Inst. Japan, ISIJ, Tokyo, (1989).

2) K. Sakaue: ISIJ Int., 30 (1990), 475.

3) T, Takeuchi: ISIJ Int., $30(1990), 482$.

4) Y. Mishima: ISIJ Int., 30 (1990), 496. 